\title{
Contribution of intraoperative stimulation mapping to glioblastoma surgery within or adjacent to descending motor pathways: survival analysis and functional outcome comparison between two series in a single institution
}

Jimmy Ming-Jung Chuang ( $\square$ jimmymjc@gmail.com )

Chang Gung Memorial Hospital Kaohsiung Branch https://orcid.org/0000-0001-5702-9937

\section{Li-Han Lin}

Kaohsiung Medical Center: Chang Gung Memorial Hospital Kaohsiung Branch

Meng-Hsiang Chen

Kaohsiung Medical Center: Chang Gung Memorial Hospital Kaohsiung Branch

Wei-Che Lin

Kaohsiung Medical Center: Chang Gung Memorial Hospital Kaohsiung Branch

Cheng-Hsien Lu

Kaohsiung Medical Center: Chang Gung Memorial Hospital Kaohsiung Branch

Yu-Jie Huang

Kaohsiung Medical Center: Chang Gung Memorial Hospital Kaohsiung Branch

Shih-Yuan Hsu

Kaohsiung Medical Center: Chang Gung Memorial Hospital Kaohsiung Branch

\section{Wu-Fu Chen}

Kaohsiung Medical Center: Chang Gung Memorial Hospital Kaohsiung Branch Jih-Tsun Ho

Kaohsiung Medical Center: Chang Gung Memorial Hospital Kaohsiung Branch

\section{Research Article}

Keywords: glioblastoma, intraoperative stimulation mapping, extent of ratio, survival analysis, functional outcome

Posted Date: March 10th, 2021

DOI: https://doi.org/10.21203/rs.3.rs-302485/v1 
License: (c) (i) This work is licensed under a Creative Commons Attribution 4.0 International License. Read Full License 


\section{Abstract}

Purpose Extensive resection probably confers a modest survival advantage in patients with glioblastoma. Studies have revealed the positive effects of intraoperative stimulation mapping (ISM) on the extent of resection, but no consensus for contribution of intraoperative stimulation mapping is reached.

Methods This retrospective study enrolled two groups of patients who underwent surgery for motoreloquent glioblastoma: the non-ISM group of 57 patients (surgery in 2008-2013) and ISM group of 13 patients (surgery in 2014-2015). The two groups and subgroups based on resection extent and postoperative additional neurological deficit were compared using Kaplan-Meier analysis and the logrank test.

Results Gross or near total resection ( $\geq 90 \%$ resection quality) was significantly more common in the ISM group than the non-ISM group $(76.9 \%$ versus $24.6 \% ; p=0.001)$. The extent of resection was also significantly greater $(90.5 \% \pm 15.6 \%$ versus $64.6 \% \pm 29.2 \% ; p=0.002)$. The neurological outcome in the ISM group was thus superior, but the two differences were not significant. The median progression-free survival time was significantly longer in the ISM group (22.0 \pm 5.1 months vs $8.0 \pm 1.0$ months; $p=0.037$ ) but a significant difference was not indicated in median overall survival time $(22.0 \pm 8.4$ months vs $16.0 \pm$ 2.2 months; $p=0.167)$.

Conclusion ISM was discovered to lead to higher quality of resection and delayed recurrence. The neurological outcome and median overall survival time in the ISM group was thus superior, but the two differences were not significant.

\section{Trial registration number (for clinical trials) Nil}

\section{Introduction}

Malignant glioma is invasive and infiltrative [1], and surgical resection is unlikely to be curative [2, 3]. In most patients, tumor recurrence occurs and survival is poor [4]. Of numerous factors related to the specific tumor and treatment employed, only patient age, performance status, some molecular markers, and tumor histology have been identified as reliable prognostic factors [5-10]. Whether resection is worthwhile in glioblastoma has long been debated, but scholars have believed that a modest survival advantage is conferred by maximal resection [11-13].

Because tumors are often located in eloquent areas, with evidence showing that involvement of these areas results in disease progression and poor survival $[14,15]$, the aim of modern surgical policy is maximization of the quality of resection and minimization of the risk of surgical sequelae [16]. Glioblastoma resections are increasingly being guided by emerging presurgical functional imaging and intraoperative mapping techniques [17]. This modern strategy has been shown to ensure the greatest resection extent and to reduce the neurological deficit caused by surgery [18]. 
However, the true contribution of the intraoperative mapping technique remains controversial. Few studies have compared use and nonuse of intraoperative stimulation mapping (ISM) for brain tumor surgery in a single institution $[19,20]$. In our department, ISM began to be used in glioma surgery in 2014. This study, conducted in two consecutive periods (2008 to 2013 and 2014 to 2015) in a single department, is a unique comparative study determining the possible contribution of intraoperative electrostimulation mapping to glioblastoma resection surgery, particularly resection within or adjacent to the descending motor pathways.

\section{Material And Methods}

\section{Patients}

This was a single institution, retrospective study of data collected as part of standard care. We identified 70 consecutive patients who underwent glioblastoma resection surgery adjacent to or within the descending motor pathways from January 2008 to December 2015. Our institute began using ISM for brain tumor surgery since Januaey 2014. The first 57 patients, who underwent conventional surgery without ISM between January 2008 and December 2013, were defined as the non-ISM group; the subsequent 13 patients, who underwent resection surgery with ISM between January 2014 and December 2015, were defined as the ISM group.

We obtained clinical data from the patients' medical records, including the patients' demographics, neuroradiologic data, intraoperative findings, postoperative outcomes, Karnofsky Performance Status (KPS) score, recurrence, and follow-up. All patients underwent baseline and volumetric magnetic resonance imaging (MRI) studies. The ISM group also underwent functional MRI (fMRI) and diffusion tensor imaging (DTI) preoperatively. In the non-ISM group, fMRI and DTI were performed prior to surgery in only five cases. The World Health Organization (WHO) brain tumor classification was employed for pathology classification [21]. Following the initial surgical resection, radiation therapy and adjuvant chemotherapy were administered to patients as the standard therapy [4].

All adult patients who underwent primary glioblastoma resection surgery within or adjacent to the descending motor pathways were included in the study based on imaging criteria for the motor eloquent cortex and diffusion tensor imaging. The exclusion criteria were tumor involving another eloquent area (visual, language, and supplementary area), bleeding with hemiplegia before surgery, and cases in which only biopsy was performed. The institutional review board of our institution approved the study protocol (IRB No.: 201800151B0).

\section{Intraoperative electrostimulation protocol}

Similar surgical equipment (neuronavigation, ultrasonic aspirator, microscope, and microinstrument) were used in the operating room for both groups, except for the intraoperative electrostimulation equipment. General anesthesia was employed in all surgeries. 
The intraoperative electrostimulation protocol was implemented using the approach of Berger and Ojemann [22]. The objective of using ISM in surgery was maximization of the amount of resection and minimization of neurological deficit due to surgical damage. The ISM protocol depended on how the tumor related to the subcortical white matter and functional cortex. Prior to resection, tumor location and boundaries were defined using a neuronavigation system in all cases. All operations were performed after fentanyl and propofol administration for total intravenous anesthesia. Muscle relaxants were administered only for intubation. The EBN IOM system (EB Neuro S.p.A, Florence, Italy) was employed to process the acquired data.

After dura opening, somatosensory evoked potential phase reversal was used to identify the central sulcus. The electrocorticogram was monitored using multichannel recording. Before tumor resection, mapping was implemented as direct cortical stimulation first with the largest current (5-20 mA) that elicited the target muscle without producing subsequent discharge. A bipolar electrode with 5 -mm-spaced tips delivered short-train (4-7 pulses) current with anodal and rectangular pulses at a frequency of $50 \mathrm{~Hz}$, interstimulus interval of $4 \mathrm{~ms}$, and pulse duration of $0.5 \mathrm{~ms}$. During tumor resection, subcortical stimulation was applied in a back and forth fashion for location of the descending motor pathways. The following subcortical stimulation protocol was employed: a train of four pulses of monopolar, cathodal, rectangular pulses at $50-\mathrm{Hz}$ frequency and $0.5-\mathrm{ms}$ duration. The current intensities applied were 5 to 10 $\mathrm{mA}$. Following the surgery, the lowest current intensity that induced a motor evoked potential response was determined.

\section{Postoperative evaluation and follow-up}

Before the surgery, neurological examinations (such as targeted muscle power level) and KPS score were reviewed. Neurological examination was performed on immediate postoperative day 1 ; another detailed examination was performed and the KPS score was recalculated after postoperative month 3 . On immediate postoperative day 1 , patients with stable or improving muscle power were placed in the favorable postoperative motor function group. Those whose muscle power was worsening were placed in the poorer postoperative motor function group. Permanent major neurological deficit was identified at the end of postoperative month 3 .

The first postoperative MRI scan was performed within 1 week after surgery. MRI scans were subsequently obtained every 3 months. Recurrence was defined according to RANO criteria. Recurrence was classified as local if located in or contiguous with the resection cavity, or $<3 \mathrm{~cm}$ from the primary tumor margins and distant when the recurrence lesion border was $>3 \mathrm{~cm}$ from a previous cavity [23]. Additional MRIs were requested in case of clinical suspicion. Patients in undistinguished progression/pseudoprogression status were continued to be followed and confirmed progressions were retrospectively registered with their initial date of suspicion as the date of progression. The patients were followed up from the time of the primary surgery for at least 72 months before December 2018.

\section{Statistical analysis}


We performed four separate statistical analyses. The demographic data of the non-ISM group was compared with that of the ISM group. Fischer's exact test or the $\chi^{2}$ test was employed to compare categorical variables. The independent $t$ and Mann-Whitney $U$ tests for parametric and nonparametric data were used to compare continuous variables. The statistical tests were all two-tailed. The ISM and non-ISM groups were assessed using Kaplan-Meier plots, and the log-rank test was used to compare the groups' progression-free and overall survival.

\section{Results}

\section{Patients, imaging, and presurgical neurological status}

A total 70 patients were enrolled in the present study. From January 2008 to December 2015, 11 surgeons participated in conventional resection without ISM in 57 patients. We began intraoperative electrostimulation mapping and monitoring for brain tumor surgery since January 2014. Between January 2014 and December 2015, 13 patients underwent resection surgery with ISM, with the procedures performed by three surgeons (who were also among the aforementioned 11 surgeons). Table 1 presents the clinical characteristics, image findings and presurgical functional status. No differences existed between the ISM and non-ISM groups in sex, age, tumor volume, preoperative neurological status, or Ki-67 index.

\section{Extent of resection and surgical sequela}

Table 2 presents surgical findings and postsurgical outcomes. GTR or NTR ( $>90 \%$ resection quality) was achieved in significantly more patients of the ISM group $(76.9 \%$ vs $24.6 \% ; p=0.001)$. Resection extent was also significantly greater in the ISM group $(90.5 \% \pm 15.6 \%$ vs $64.6 \% \pm 29.2 \% ; p=0.002)$.

Immediate postoperative additional neurological deficit was half as common in the ISM group (15.4\% vs $28.1 \%$ ). In the ISM group, permanent major neurological deficit was also less common than that in the non-ISM group (7.7\% vs $21.1 \%$ ). The neurological outcome in the ISM group was thus superior, but the two differences were not significant.

\section{Survival analysis}

In the survival analysis comparing the ISM and non-ISM groups, the median progression-free survival time was significantly longer in the ISM group (22.0 \pm 5.1 months vs $8.0 \pm 1.0$ months; $p=0.037)$. Also, the median overall survival time was longer in the ISM group ( $22.0 \pm 8.4$ months vs $16.0 \pm 2.2$ months; $p$ $=0.167)$, but this difference was nonsignificant. The progression-free and overall survival Kaplan-Meier curves are displayed in Fig. 1a and b, respectively.

In the GTR + NTR versus SR + PR survival analysis comparison, the median progression-free survival time was significantly longer in the $\geq 90 \%$ resection group ( $16.0 \pm 5.3$ months vs $8.0 \pm 1.4$ months; $p=0.018$ ). The median overall survival time was also significantly longer in the $\geq 90 \%$ resection group $(37.0 \pm 9.2$ 
months vs $14.0 \pm 2.8$ months; $p<0.001)$. The progression-free and overall survival Kaplan-Meier curves are presented in Fig. 2a and b.

\section{Discussion}

\section{Comparative study in a single institution}

The standard treatment for patients with newly diagnosed glioblastoma (WHO grade IV) is maximal surgical resection combined with radiotherapy plus adjuvant and concomitant temozolomide or carmustine wafers (Gliadel) [4]. Increasing evidence indicates an independent correlation between overall survival and the extent of glioblastoma resection [5, 16, 24-32]. Extensive resection in patients with glioblastoma is generally believed to confer a slight survival advantage.

However, complete resection of glioblastoma is impossible because it is invasive and infiltrative [1]. The resection of glioblastoma located adjacent to or within the eloquent cortex or a subcortical pathway structure remains difficult for surgeons [15]. In modern glioblastoma surgery, maximizing the resection extent and minimizing the risk of surgical sequelae are the objectives [16]. Surgery safety and resection extents have been increased by advances such as neuronavigation, fMRI [33,34], intraoperative MRI [35], fluorescence-guided surgery [24], laser interstitial thermal therapy [36], and intraoperative electrostimulation mapping [37].

The recent literature reveals that the use of ISM during tumor surgery in eloquent areas has a positive effect; however, controversy remains. No randomized trials have yet been conducted on the usefulness of ISM. Because of the multiple advanced tools employed in tumor resection, assessment of ISM's exact role is difficult. Additionally, different institutions usually employ differing surgical strategies and equipment. Furthermore, achieving homogeneity in tumor histology and location is difficult in a study. Only two comparative studies have been reported in the literature regarding operation without and with ISM for brain tumor in a single institution $[19,20]$. Reithmeier et al. [19] included patients with low-grade to high-grade gliomas and even metastasis and meningioma. Duffau et al. [20] published a series of landmark articles but focused on supratentorial low-grade glioma and the multiple locations of these tumors.

To minimize the aforementioned bias, our study is the first comparative study conducted in a single institution that compares the possible contribution of ISM to glioblastoma resection surgery and with a focus on tumors adjacent to or within the descending motor pathways. Our sample was uniform and consecutive in an attempt to determine the effect of ISM on resection quality, functional outcome, and survival.

\section{Contribution of ISM to resection quality and survival analysis}

Our study revealed that $>90 \%$ resection quality was achieved in significantly more cases and the resection extent was significantly greater in the ISM group than the non-ISM group. Gerritsen et al. [38] published a 
meta-analysis investigating the effect of ISM in patients with high-grade glioma. GTR was significantly more prevalent in the ISM group than the non-ISM group $(79.1 \%$ vs $47.7 \% ; p<0.001)$. However, the comparison performed in this meta-analysis was subject to considerable publication bias, selection bias, and poor uniformity of tumor information [18]. Thus, in the absence of level I data, the present study must be considered a pilot study obtaining proof that use of ISM has a significant impact on resection quality.

Several studies conducted in the last 20 years have furthered our understanding of the importance of resection extent for low-grade and high-grade glioma [16, 24-32]. Brown et al. [32] performed the largest quantitative meta-analysis and discovered that compared with SR, GTR had a strong positive effect on overall and progression-free survival. Stummer et al. [24] performed the prospective, randomized trials for use of 5-ALA that have shown survival advantage of gross total resection. This was also shown in our study; the group with $\geq 90 \%$ resection (GTR + NTR) had longer overall and progression-free survival than those with $<90 \%$ resection. However, in the survival analysis comparing the ISM and non-ISM groups, the median progression-free survival time was significantly longer in the ISM group ( $22.0 \pm 5.1$ months vs 8.0 \pm 1.0 months; $p=0.037$ ), and a significant difference was not indicated in median overall survival time $(22.0 \pm 8.4$ months vs $16.0 \pm 2.2$ months; $p=0.167)$. There are two possible reasons: the resection quality was favorable in some cases in the non-ISM group, or an extent of resection threshold-the lowest amount of resection that confers a survival benefit in glioblastoma surgery-was not met in the ISM group. This resection threshold has been reported as between 70\% and 98\% [5, 39-42]. Therefore, resection with use of ISM must be sufficiently large to confer a survival benefit in glioblastoma surgery.

\section{Contribution of ISM to functional outcome and survival analysis}

In our study, a postoperative immediate additional neurological deficit was almost half as common in the ISM group compared with the non-ISM group (15.4\% vs $28.1 \%)$. A permanent major neurological deficit was also less common in the ISM group (7.7\% vs $21.1 \%)$. The neurological outcome in the ISM group was thus superior, but the difference in both postoperative immediate additional neurological deficit and permanent major neurological deficit were nonsignificant. De Witt et al. [18] conducted a landmark systematic meta-analysis on the safety of resective glioma surgery. They reported that both early neurologic deficits (14.2\% vs $47.9 \%)$ and late neurologic deficits $(6.4 \%$ vs $9.4 \%)$ were less common in the ISM group.

Intraoperative electrostimulation can create a safe distance to critical functional structures by adjusting the stimulation current or striking the resection cavity wall with the stimulator to prevent direct extensive parenchymal damage. However, this is not always sufficient to avoid vascular damage in particular, which causes irreversible ischemia and delayed hypoperfusion. Permanent neurological deficits have usually been related to inadvertent surgical ischemia, which is detectable using postoperative diffusionweighted MRI $[43,44]$.

\section{Limitations of our study}


Our study was limited in that it had a retrospective design and variability may have been caused by the bias of the attending surgeons. Additionally, the sample was small, and this was a single-center study. Another crucial bias, especially for overall survival, was the lack of molecular pathological data.

Treatment of glioblastoma through surgery alone is difficult. Tumor recurrence occurs in the majority of patients because of the low extent of resection but also the molecular subtype of glioblastoma. This is why greater resection extent resulted in longer median progression-free survival but not longer overall survival.

\section{Conclusion}

This is the first uniform and comparative analysis investigating the contribution of ISM in a single institution for treating glioblastoma in motor-eloquent area. Our results revealed that the use of ISM led to higher quality of resection and delayed recurrence. Although the effect on postoperative functional outcome and overall survival was nonsignificant. ISM may be useful as a tool in glioblastoma surgery but controversial to be a necessity for its contribution.

\section{Declarations}

Patient consent This retrospective research is conducted on electronic medical record from Kaohsiung Chang Gung Memorial Hospital. Therefore, only records in existence at the time of IRB review and approval will be accessed for review. A waiver of consent will be sought from the IRB as re-contacting this number of patients to obtain informed consent would be impracticable and would hinder our ability to conduct the study.

IRB approval This retrospective study was approved by Chang Gung Memorial Hospital's Institutional Review Committee on Human Research (IRB No.: 201800151B0).

Provenance and peer review Commissioned; externally peer reviewed.

Acknowledgment This research was supported by Kaohsiung Chang Gung Memorial Hospital Neurooncology Multidisciplinary Team. We thank our colleagues who provided insight and expertise that greatly assisted the research, although they may not agree with all of the interpretations/conclusions of this paper.

\section{ORCID IDs}

Jimmy Ming-Jung Chuang https://orcid.org/0000-0001-5702-9937

Meng-Hsiang Chen https://orcid.org/0000-0003-0201-9015

Wei-Che Lin https://orcid.org/0000-0002-1326-0587

Wu-Fu Chen https://orcid.org/0000-0002-6910-2692 
Funding statement or sources of support The authors have not declared a specific grant for this research from any funding agency in the public, commercial or not-for-profit sectors.

Competing interests All authors declare neither financial nor non-financial interests that may be relevant to the submitted work.

Data availability statement The data that support the findings of this study are available from the corresponding author upon reasonable request.

Code availability Data available on request

Author contribution Conceptualization: [Dr. Jimmy Ming-Jung Chuang]; Methodology: [Dr. Jimmy MingJung Chuang, Dr. Jih-Tsun Ho]; Formal analysis and investigation: [Dr. Jimmy Ming-Jung Chuang, Dr. LiHan Lin, Meng-Hsiang Chen, Dr. Yu-Jie Huang, Shih-Yuan Hsu]; Writing - original draft preparation: [Dr. Jimmy Ming-Jung Chuang]; Writing - review and editing: [Dr. Cheng-Hsien Lu], Supervision: [Dr. Jih-Tsun Ho]; Critical revision of the manuscript for important intellectual content: [Dr. Wei-Che Lin, Dr. Wu-Fu Chen, Dr. Cheng-Hsien Lu]

Ethical approval This retrospective study was approved by Chang Gung Memorial Hospital's Institutional Review Committee on Human Research.

Consent to participate This retrospective research is conducted on electronic medical record from Kaohsiung Chang Gung Memorial Hospital. Therefore, only records in existence at the time of IRB review and approval will be accessed for review. A waiver of consent will be sought from the IRB as re-contacting this number of patients to obtain informed consent would be impracticable and would hinder our ability to conduct the study.

Consent for publication I give my consent for the publication of identifiable details, which can include tables and figures within the text in the above article. I have discussed with all the co-authers of this paper.

\section{References}

1. Claes A, Idema AJ, Wesseling P (2007) Diffuse glioma growth: a guerilla war. Acta Neuropathol 114:443-458. https://dx.doi.org/10.1007\%2Fs00401-007-0293-7

2. Chang $\mathrm{CH}$, Horton J, Schoenfeld D et al (1983) Comparison of postoperative radiotherapy and combined postoperative radiotherapy and chemotherapy in the multidisciplinary management of malignant gliomas. A joint radiation therapy oncology group and eastern cooperative oncology group study. Cancer 52:997-1007. https://doi.org/10.1002/1097-0142(19830915)52:6\%3C997::AIDCNCR2820520612\%3E3.0.CO;2-2 
3. Walker MD, Alexander E Jr, Hunt WE et al (1978) Evaluation of BCNU and/or radiotherapy in the treatment of anaplastic gliomas: a cooperative clinical trial. J Neurosurg 49:333-343. https://doi.org/10.3171/jns.1978.49.3.0333

4. Wen PY, Kesari S (2008) Malignant gliomas in adults. N Engl J Med 359:492-507. https://doi.org/10.1056/NEJMra0708126

5. Lacroix M, Abi-Said D, Fourney DR et al (2001) A multivariate analysis of 416 patients with glioblastoma multiforme: prognosis, extent of resection, and survival. J Neurosurg 95:190-198. https://doi.org/10.3171/jns.2001.95.2.0190

6. Cohen AL, Holmen SL, Colman H (2013) IDH1 and IDH2 mutations in gliomas. Curr Neurol Neurosci Rep 13:345. https://doi.org/10.1056/NEJMoa0808710

7. Aldape K, Zadeh G, Mansouri S et al (2015) Glioblastoma: pathology, molecular mechanisms and markers. Acta Neuropathol 129:829-848. https://doi.org/10.1007/s00401-015-1432-1

8. Pelloski CE, Ballman KV, Furth AF et al (2007) Epidermal growth factor receptor variant III status defines clinically distinct subtypes of glioblastoma. J Clin Oncol 25:2288-2294. https://doi.org/10.1200/jco.2006.08.0705

9. Hegi ME, Diserens AC, Gorlia T et al (2005) MGMT gene silencing and benefit from temozolomide in glioblastoma. N Engl J Med 352:997-1003. https://doi.org/10.1056/nejmoa043331

10. Juratli TA, Kirsch M, Geiger $\mathrm{K}$ et al (2012) The prognostic value of IDH mutations and MGMT promoter status in secondary high-grade gliomas. J Neurooncol 110:325-333. https://doi.org/10.1007/s11060-012-0977-2

11. Hart MG, Metcalfe SE, Grant R (2000) Biopsy versus resection for high grade glioma. Cochrane Database Syst Rev 2000:CD002034. https://dx.doi.org/10.1002\%2F14651858.CD002034

12. Proescholdt MA, Macher $C$, Woertgen $C$ et al (2005) Level of evidence in the literature concerning brain tumor resection. Clin Neurol Neurosurg 107:95-98. https://doi.org/10.1016/j.clineuro.2004.02.025

13. Sawaya R (1999) Extent of resection in malignant gliomas: a critical summary. J Neurooncol 42:303305. https://doi.org/10.1023/a:1006167412835

14. Chang EF, Clark A, Smith JS et al (2011) Functional mapping-guided resection of low-grade gliomas in eloquent areas of the brain: improvement of long-term survival. J Neurosurg 114:566-573. https:// doi.org/10.3171/2010.6.jns091246

15. Skirboll SS, Ojemann GA, Berger MS et al (1996) Functional cortex and subcortical white matter located within gliomas. Neurosurgery 38:678-685. https://doi.org/10.1227/00006123-19960400000008

16. Hervey-Jumper SL, Berger MS (2016) Maximizing safe resection of low- and high-grade glioma. J Neurooncol 130:269-282. https://doi.org/10.1007/s11060-016-2110-4

17. Sanai N, Berger MS (2010) Intraoperative stimulation techniques for functional pathway preservation and glioma resection. Neurosurg Focus 28:E1. https://doi.org/10.3171/2009.12.focus09266 
18. De Witt Hamer PC, Robles SG, Zwinderman AH et al (2012) Impact of intraoperative stimulation brain mapping on glioma surgery outcome: a meta-analysis. J Clin Oncol 30:2559-2565. https://doi.org/10.1200/jco.2011.38.4818

19. Reithmeier T, Krammer M, Gumprecht $\mathrm{H}$ et al (2003) Neuronavigation combined with electrophysiological monitoring for surgery of lesions in eloquent brain areas in 42 cases: a retrospective comparison of the neurological outcome and the quality of resection with a control group with similar lesions. Minim Invasive Neurosurg 46:65-71. https://doi.org/10.1055/s-200339334

20. Duffau H, Lopes $M$, Arthuis $F$ et al (2005) Contribution of intraoperative electrical stimulations in surgery of low grade gliomas: a comparative study between two series without (1985-96) and with (1996-2003) functional mapping in the same institution. J Neurol Neurosurg Psychiatry 76:845-851. https://doi.org/10.1136/jnnp.2004.048520

21. Louis DN, Ohgaki H, Wiestler OD et al (2007) The 2007 WHO classification of tumours of the central nervous system. Acta Neuropathol 114:97-109. https://dx.doi.org/10.1007\%2Fs00401-007-0243-4

22. Berger MS, Ojemann GA (1992) Intraoperative brain mapping techniques in neuro-oncology. Stereotact Funct Neurosurg 58:153-161. https://doi.org/10.1159/000098989

23. Wen PY, Macdonald DR, Reardon DA et al (2010) Updated response assessment criteria for highgrade gliomas: response assessment in neuro-oncology working group. J Clin Oncol 28:1963-1972. https://doi.org/10.1200/jco.2009.26.3541

24. Stummer W, Pichlmeier U, Meinel T et al (2006) Fluorescence-guided surgery with 5-aminolevulinic acid for resection of malignant glioma: a randomised controlled multicentre phase III trial. Lancet Oncol 2006;7:392-401. https://doi.org/10.1016/s1470-2045(06)70665-9

25. Sanai N, Berger MS (2008) Glioma extent of resection and its impact on patient outcome. Neurosurgery 62:753-764. https://doi.org/10.1227/01.neu.0000318159.21731.cf

26. McGirt MJ, Chaichana KL, Gathinji M et al (2009) Independent association of extent of resection with survival in patients with malignant brain astrocytoma. J Neurosurg 110:156-162. https://doi.org/10.3171/2008.4.17536

27. Almeida JP, Chaichana KL, Rincon-Torroella J et al (2015) The value of extent of resection of glioblastomas: clinical evidence and current approach. Curr Neurol Neurosci Rep 15:517. https://doi.org/10.1007/s11910-014-0517-x

28. Keles GE, Chang EF, Lamborn KR et al (2006) Volumetric extent of resection and residual contrast enhancement on initial surgery as predictors of outcome in adult patients with hemispheric anaplastic astrocytoma. J Neurosurg 105:34-40. https://doi.org/10.3171/jns.2006.105.1.34

29. Smith JS, Chang EF, Lamborn KR et al (2008) Role of extent of resection in the long-term outcome of low-grade hemispheric gliomas. J Clin Oncol 26:1338-1345. https://doi.org/10.1200/jco.2007.13.9337

30. Vecht CJ, Avezaat CJ, van Putten WL et al (1990) The influence of the extent of surgery on the neurological function and survival in malignant glioma. A retrospective analysis in 243 patients. $J$ 
Neurol Neurosurg Psychiatry 53:466-471. https://doi.org/10.1136/jnnp.53.6.466

31. Senft C, Bink A, Franz K et al (2011) Intraoperative MRI guidance and extent of resection in glioma surgery: a randomised, controlled trial. Lancet Oncol 12:997-1003. https://doi.org/10.1016/s14702045(11)70196-6

32. Brown TJ, Brennan MC, Li M et al (2016) Association of the extent of resection with survival in glioblastoma: a systematic review and meta-analysis. JAMA Oncol 2:1460-1469. https://doi.org/10.1001/jamaoncol.2016.1373

33. Trinh VT, Fahim DK, Maldaun MV et al (2014) Impact of preoperative functional magnetic resonance imaging during awake craniotomy procedures for intraoperative guidance and complication avoidance. Stereotact Funct Neurosurg 92:315-322. https://doi.org/10.1159/000365224

34. Guggisberg AG, Honma SM, Findlay AM et al (2008) Mapping functional connectivity in patients with brain lesions. Ann Neurol 63:193-203. https://doi.org/10.1002/ana.21224

35. Wirtz CR, Knauth M, Staubert A et al (2000) Clinical evaluation and follow-up results for intraoperative magnetic resonance imaging in neurosurgery. Neurosurgery 46:1112-1122. https://doi.org/10.1097/00006123-200005000-00017

36. Missios S, Bekelis K, Barnett GH (2015) Renaissance of laser interstitial thermal ablation. Neurosurg Focus 38:E13. https://doi.org/10.3171/2014.12.focus14762

37. Asthagiri AR, Pouratian N, Sherman J et al (2007) Advances in brain tumor surgery. Neurol Clin 2007;25:975-1003. https://doi.org/10.1016/j.ncl.2007.07.006

38. Gerritsen JKW, Arends L, Klimek M et al (2019) Impact of intraoperative stimulation mapping on high-grade glioma surgery outcome: a meta-analysis. Acta Neurochir (Wien) 161:99-107. https://doi.org/10.1007/s00701-018-3732-4

39. Sanai N, Polley MY, McDermott MW et al (2011) An extent of resection threshold for newly diagnosed glioblastomas. J Neurosurg 115:3-8. https://doi.org/10.3171/2011.2.jns10998

40. Orringer D, Lau D, Khatri $S$ et al (2012) Extent of resection in patients with glioblastoma: limiting factors, perception of resectability, and effect on survival. J Neurosurg 117:851-859. https://doi.org/10.3171\%2F2012.8.JNS12234

41. Chaichana KL, Jusue-Torres I, Navarro-Ramirez R et al (2014) Establishing percent resection and residual volume thresholds affecting survival and recurrence for patients with newly diagnosed intracranial glioblastoma. Neuro Oncol 16:113-122. https://doi.org/10.1093/neuonc/not137

42. Grabowski MM, Recinos PF, Nowacki AS et al (2014) Residual tumor volume versus extent of resection: predictors of survival after surgery for glioblastoma. J Neurosurg 121:1115-1123. https://doi.org/10.3171/2014.7.jns132449

43. Smith JS, Cha S, Mayo MC et al (2005) Serial diffusion-weighted magnetic resonance imaging in cases of glioma: distinguishing tumor recurrence from postresection injury. J Neurosurg 103:428438. https://doi.org/10.3171/jns.2005.103.3.0428

44. Ulmer S, Braga TA, Barker FG et al (2006) Clinical and radiographic features of peritumoral infarction following resection of glioblastoma. Neurology 67:1668-1670. 
https://doi.org/10.1212/01.wnl.0000242894.21705.3c

\section{Tables}

Table 1 Comparisons of clinical features, image findings and presurgical functional status between patients with GBM adjacent to motor-eloquent area following non-ISM and ISM method

\begin{tabular}{|lllr|}
\hline Sex (Male/female) & $35 / 22$ & $8 / 5$ & 1.000 \\
\hline Mean age at onset & $57.32 \pm 14.51$. & $59.69 \pm 12.79$ & 0.563 \\
\hline Media MP on presentation & $4(0-5)$ & $4(2-5)$ & 0.923 \\
\hline Mean KPS on presentation & $69.12 \pm 9.69$ & $73.08 \pm 11.09$ & 0.252 \\
\hline Neuroimaging findings at presentation & & & \\
\hline Left/Right hemisphere & $18 / 39$ & $5 / 8$ & 0.746 \\
\hline Mean Volume of brain tumor $\left(\mathrm{mm}^{3}\right)$ & $52856.49 \pm 34274.70$ & $37165.80 \pm 18970.57$ & 0.116 \\
\hline
\end{tabular}

Table 2. Comparisons of surgical findings and postsurgical outcomes between patients with GBM adjacent to motor-eloquent area following non-ISM and ISM method

\begin{tabular}{|llll|}
\hline Surgical findings & & & \\
\hline GTR or NTR/Subtotal & $14 / 43$ & $10 / 3$ & 0.001 \\
\hline Extent of Resection (\%) & $64.55 \pm 29.17$ & $90.51 \pm 15.58$ & 0.002 \\
\hline Mean Volume of residual tumor $\left(\mathrm{mm}^{3}\right)$ & $\begin{array}{l}18699.33 \pm \\
20939.45\end{array}$ & $\begin{array}{c}3957.52 \pm \\
4594.99\end{array}$ & 0.014 \\
\hline Estimated Blood Loss & $391.58 \pm 287.18$ & $263.85 \pm 309.69$ & 0.192 \\
\hline Functional Outcome & & & 0.491 \\
\hline $\begin{array}{l}\text { Stationary or Improving/ Worse (Post-op day } \\
\text { ) }\end{array}$ & $41 / 16$ & $11 / 2$ & 0.437 \\
\hline $\begin{array}{l}\text { Major Neurological Deficit (Post-op 3 } \\
\text { months) }\end{array}$ & 12 & 1 & \\
\hline $\begin{array}{l}\text { Pathology } \\
\text { Ki-67(\%) }\end{array}$ & $31.09 \pm 17.75$ & $38.69 \pm 21.84$ & 0.259 \\
\hline
\end{tabular}

\section{Figures}


A

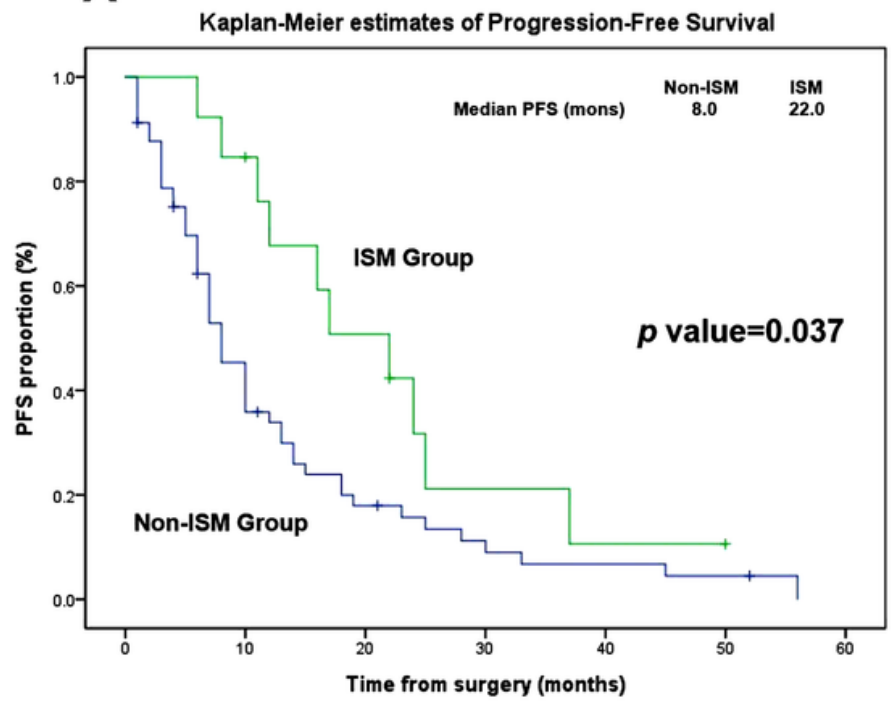

B

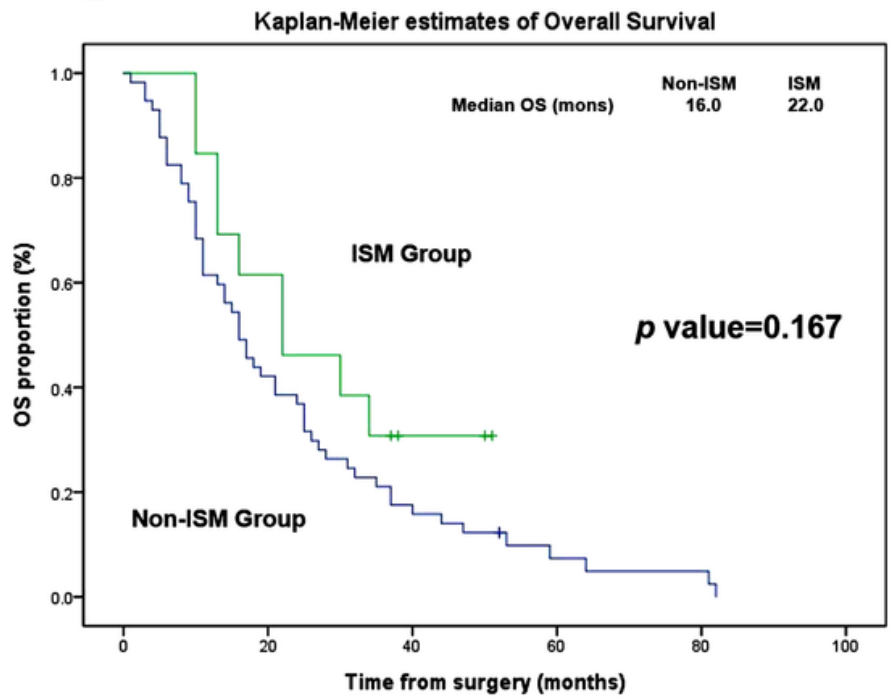

Figure 1

Kaplan-Meier survival curve of a progression-free survival and b overall survival in ISM and non-ISM groups (ISM intraoperative stimulation mapping, PFS progression-free survival, OS overall survival)

A

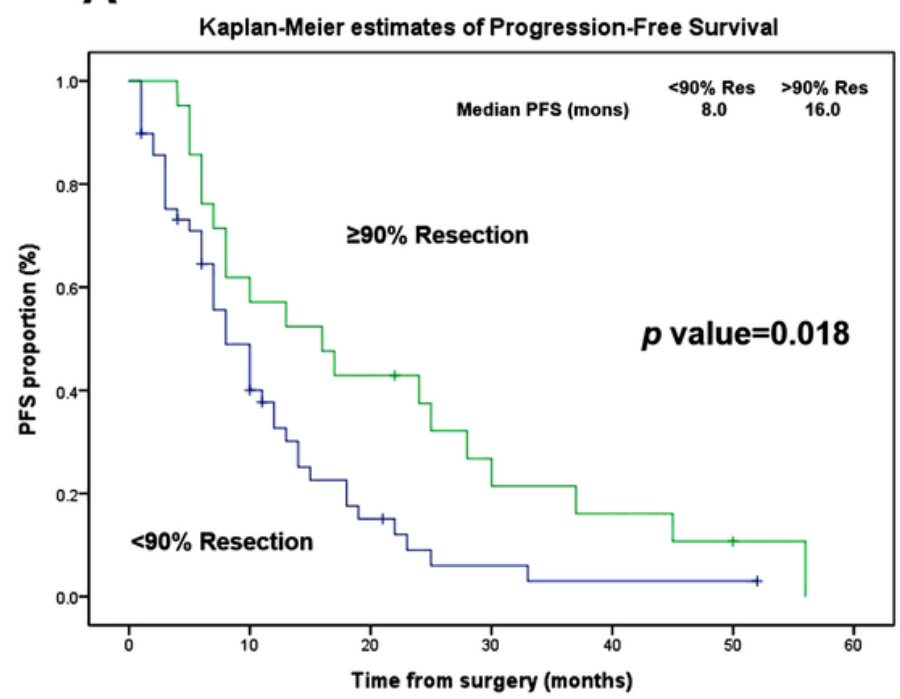

B

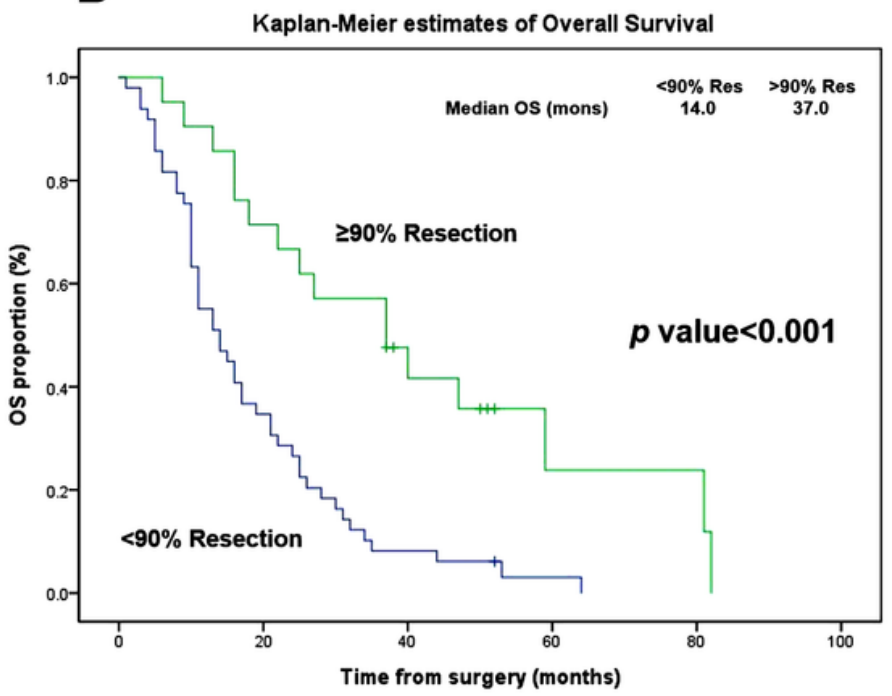

Figure 2

Kaplan-Meier survival curves of a progression-free survival and b overall survival in the groups with $\geq 90 \%$ resection and $<90 \%$ resection 